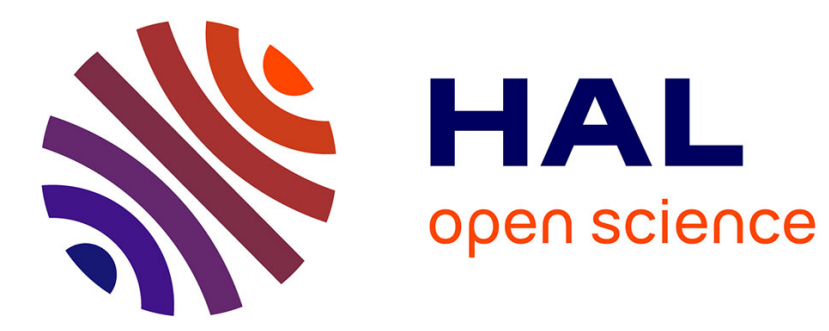

\title{
Étude spectrale du groupe de raies telluriques nommé $\alpha$ (alpha) par Angström
}

\author{
A. Cornu
}

\section{To cite this version:}

A. Cornu. Étude spectrale du groupe de raies telluriques nommé $\alpha$ (alpha) par Angström. J. Phys. Theor. Appl., 1884, 3 (1), pp.109-117. 10.1051/jphystap:018840030010900 . jpa-00238183

\section{HAL Id: jpa-00238183 https://hal.science/jpa-00238183}

Submitted on 1 Jan 1884

HAL is a multi-disciplinary open access archive for the deposit and dissemination of scientific research documents, whether they are published or not. The documents may come from teaching and research institutions in France or abroad, or from public or private research centers.
L'archive ouverte pluridisciplinaire HAL, est destinée au dépôt et à la diffusion de documents scientifiques de niveau recherche, publiés ou non, émanant des établissements d'enseignement et de recherche français ou étrangers, des laboratoires publics ou privés. 


\title{
ÉTUDE SPEGTRALE DU GROUPE DE RAIES TELLURIQUES NOMMÉ $\propto$ (ALPHA) PAR ANGSTRÖM;
}

\author{
PAR M. A. GORNU.
}

Depuis les travaux de Brewster et de M. Janssen, on sait que le spectre solaire présente des bandes ou groupes de raies sombres d'intensité variable avec la chaleur du Soleil, produites par l'absorption due à l'atmosphère terrestre. Ces principaux groupes telluriques ont reçu des désignations particulières : à l'extrême rouge, la raie $\mathrm{A}$ de Fraunhofer est d'origine atmosphérique; il en est de niême du groupe $a$ et de la raie $B$; plus loin, entre $C$ et $D$, se trouvent d'abord un groupe moins intense nommé $\alpha$ par Angström, puis les raies telluriques voisines de $\mathrm{D}$, dont j'ai donñé récemment une description spéciale (Journal de Physique, $2^{\text {e }}$ série, t. II, p. 58); enfin, au delà de la raie $\mathrm{D}$, une bande $\delta$ (Brewster) termine la série des principales bandes atmosphériques du spectre visible.

La figure ci-dessous indique la répartition des raies telluriques dans le spectre solaire visible : d'après nos connaissances actuelles,

Fig. I.

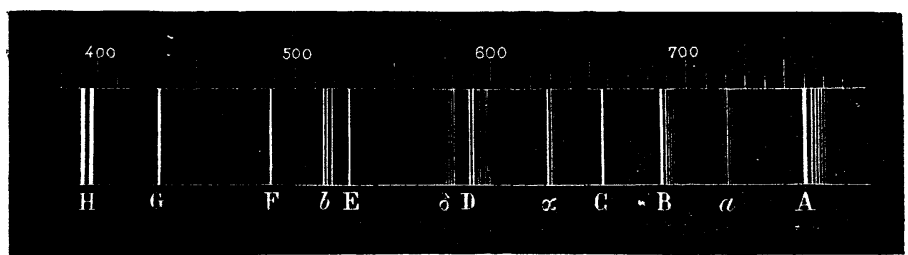

les groupes $\mathrm{A}, \mathrm{B}, \alpha$ et ò seraient dus à l'atmosphère sèche : les groupes $a$ et ceux voisins de C et de D seraient causés par la vapeur d'eau.

La continuation de mes études sur les raies telluriques des régions les plus lumineuses du spectre solaire m`a conduit naturellement à examiner en détail le groupe $\alpha$, découvert par Brewster, situé dans l'orangé, entre les raies C et D de Fraunhofer. Ce groupe, d'apparence complexe (1), mérite une attention toute spé-

(') Voir Hadeira spectroscopic, par M. C. Piazzi Smyth, et I'Mllas de M. Nívez. J. cle I'hy's., ze séric, t. III. ( Mars r $88 \%$.) 
ciale; d'abord il devient extrêmement intense lorsque le Soleil s'approche de l'horizon, ce qui dénole dans l'atmosphère terrestre la présence d'un élément doué d'un pouvoir absorbant considérable et dont l'étude s'impose nécessairement. Angström a depuis longtemps reconnu que la substance absorbante qui le produil n'est pas la vapeur d'eau, car il persiste aux plus grands froids des hivers de Suède. En second lieu, ce groupe, bien que situé dans une région déjà assez sombre, reste très visible, même avec une forte dispersion, lorsque le Soleil atteint l'horizon : l'étude peut donc en être suivie à toute époque de la journée.

L'Atlas d'Angström est malheureusement assez incomplet dans cette région : il a donc fallu commencer par refaire la carte de ce groupe en adoptant une échelle quatre ou cinq fois plus grande. Les premières journées d'observation (août et septembre i 883 , Courtenay, Loiret) se passèrent à relever les raies visibles et à noter leurs variations relatives avec la hauteur du Soleil. Comme mes devanciers, je ne vis d'abord qu'un amas complexe de lignes sombres, distribuées irrégulièrement comme les raies telluriques voisines de $\mathrm{D}$; mais de cette confusion sortit tout à coup une régularité singulière lorsque je parvins à démêler avec certitude les diverses espèces de raies qui se superposent dans ce groupe et qui appartiennent au moins à trois catégories distinctes. Il n'est peutêtre pas sans intérêt d'indiquer brièvement la marche suivie pour atteindre ce résultat.

$I^{\circ}$ Raies d'origine solaire. - Avec le réseau Rutherfurd dont je faisais d'abord usage (Comptes rendus, t. XGV, p. 8o I), la distinction des raies telluriques d'avec les raies solaires se faisait par la considération des intensités relatives à diverses hauteurs du Soleil. Cette détermination est extrêmement longue et pénible : elle exige des séries d'observations de plusicurs heures faites dans des conditions météorologiques à peu près constantes, et les résultats ne sont décisifs que si les raies à comparer sont voisines comme distance et comme intensité. Ayant reçu de M. le professeur Rowland un magnifique réseau sur métal que l'Université John Hopkins de Baltimore a gracieusement offert à l'École Polytechnique, j'essayai une méthode permettant d'abréger le travail et de déterminer, à la simple inspection, les raies d'origine solaire. Grâce à la dispersion 
des spectres, à l'intensité et à la perfection des images de cet admirable réseau ( 1 ), la réussite a été complète.

I e principe de la méthode esı le déplacement des raies dans le spectre de la lumière émise par une source en mouvement absolu ou relatif, principe énoncé pour la première fois en I 848 par notre illustre Confrère M. Fizeau, dans un Mémoire rectifiant les idées de Doppler $\left({ }^{2}\right)$. Ce déplacement des raies spectrales a été depuis considéré bien des fois par divers spectroscopistes (Secchi, Huggins, Zöllner, etc.), mais c'est à M. Thollon qu'on doit l'expérience où le déplacement dû à la rotation du Soleil a été mis hors de doute : l'artifice imaginé par $\mathrm{M}$. Thollon consiste à comparer à des raies telluriques fixes les raies solaires, qui se déplacent suivant qu'on prend comme source l'une ou l'autre extrémité de l'équateur solaire (Comptes rendus, t. XCI, p. 369). Par cette observation différentielle l'auteur s'est mis à l'abri des effets de parallaxes aux.-

( $\left.{ }^{1}\right)$ La surface striée ne mesure pas moins de $0^{\mathrm{m}}, 06$ (longueur des traits) sur $0^{\mathrm{m}}, 08$ de largeur : la distance constante des traits est égale à $0^{\mathrm{mm}}, 00 \mathbf{I}_{7} 60$. L'appareil d'observation se compose d'un collimateur de $\mathrm{I}^{\mathrm{m}}, \mathrm{r} 8$ de longueur focale $\left(0^{\mathrm{m}}, 09^{\circ}\right.$ d'ouverture) et d'une lunette de $\mathrm{r}^{\mathrm{m}}$, $\mathrm{y}_{\mathrm{O}}$ ( 0,105 d'ouverture); l'angle des axes optiques est fixe (environ $42^{\circ}$ ) de manière à observer le deuxième spectre sous une incidence presque normalc.

$\left({ }^{2}\right)$ Bulletin de la Société philomathique, 23 décembre 1848 , et Annales de Chimie et de Physique, '́t série, t. IIX, p. 2 I

D'après la formule de M. Fizeau, on a

$$
\lambda^{\prime}=\lambda\left(\mathrm{r}-\frac{v}{\mathrm{v}}\right)
$$

où $\lambda$ est la longueur d'onde de la radiation émise par la source supposée au repos, $\lambda^{\prime}$ la longueur d'onde observée, $v$ la composante de la vitesse relative de la source par rapport à l'observateur suivant la direction du faisceau reçu et $\mathbf{V}$ la vitesse de la lumière $\left(30000^{\mathrm{km}}\right.$ à la seconde).

Dans le cas où l'on considère comme source le contour apparent du Soleil aux extrémités de l'équateur, le rapport $\frac{v}{V}= \pm \frac{2}{300000}$, car la vitesse d'un point de l'équateur solaire est égale très scnsiblement à $2^{\mathrm{hm}}$ par seconde. [ Le rayon solaıre équatorial égale 108,6 rayons terrestres de $6378^{\mathrm{hm}}$ et la durée de rotation du Soleil cst de vingt-cinq jours quatre heures et vingt-neuf minutes (Annuaire du Bureau des Longitudes, 3884 )]. On en conclut aisément que le déplacement des raies spectrales, dans le voisinage des raies $D_{1}, D_{2}\left(\lambda_{1}=589,4^{\circ}, \lambda_{2}=588,89\right)$, est environ $\pm \frac{1}{150}$ de la distance de ces deux raies, suivant qu'on prend comme source le bord oriental ou le bord occidental de l'équateur solaire: le déplaccment atteint donc $\frac{1}{75}$ de cette distance. Les mesures micrométriques vérifient ce calcul à l'approximation, nécessairement assez restreintc, que donnent les pointés. 
quels les dispositifs employés jusqu'ici pour ces mesures peuvent donner lieu.

L'analyse des conditions optiques de la formation des images m'a permis de faire faire à la question un pas de plus et de rendre absolue l'observation relative que $\mathrm{M}$. Thollon a si bien utilisée à la démonstration du principe de M. Fizeau.

Voici comment on doit opérer : on projette sur la fente du collimateur l'image très petite du disque solaire produite par une lentille achromatique de $\mathrm{o}^{\mathrm{m}}$, $\mathrm{r} \mathrm{o}$ à $\mathrm{o}^{\mathrm{m}}$, , $\mathrm{I} 2$ de distance focale $:$ on fait osciller rapidement (deux à trois fois par seconde) la lentille, de manière à amener alternativement les deux bords du disque tangentiellement au même point de la fente. On voit alors les raies d'origine solaire osciller comme la lentille, tandis que les raies telluriques restent fixes : les grains de poussière dont le fil horizontal du réticule est toujours parsemé servent de repères très commodes. L'amplitude de ce balancement des raies mobiles est d'autant plus grande que la direction de l'équateur solaire est plus près d'être normale à la fente; mais, grâce à l'oscillation rapide qu'on donne à l'image, le balancement est si net qu'il est reconnaissable à peu près dans toutes les orientations de l'équateur solaire. Au moindre balancement, il se produit même une singulière illusion : les raies mobiles paraissent se détacher en relief et osciller en avant du plan des raies fixes.

L'emploi d'une très petite image solaire en oscillation rapide( ' $)$ constitue déjà un perfectionnement notable à la méthode ordinai-

( $\left.{ }^{1}\right)$ Le balancement des raies est tellement favorable à la netteté de l'impression physiologique qu'il rend visibles des déplacements qui passeraient inaperçus sans cet artifice : on s'en convainc en opérant statiquement; à cet effet, on interpose entre la fente et la lentille collectrice un prisme biréfringent qui permet de rendre tangents, normalement à la fente, les deux bords opposés du disque solaire. Les raies telluriques restent rectilignes, les raies solaires sont inclinées et brisées. La brisure qui caractérise la double déviation n'est bien nette que lorsque l'orientation du disque solaire est voisine du maximum d'effet. Il est juste d'ajouter que ce mode opératoire diminue au moins de moitié l'intensité générale du champ de vision.

Néanmoins, c'est celui qu'on doit appliquer à la distinction des raies telluriques et solaires par la photographie dans les régions infra-rouge ou ultra-vıolette. Il est alors utile d'employer le dispositif bien connu du prisme à réflexion totale pour orienter l'équateur solaire dans la direction la plus favorable, c'est-à-dire parallèlement à la fente du collimateur. Des essais préliminaires m'ont prouvé qu'on 
rement employée; mais il reste à indiquer les conditions que doivent remplir les appareils, conditions sans lesquelles le phénomène perd sa netteté et se réduit à l'apparcnce différentielle de M. Thollon, au lieu d'offrir le phénomène absolu qu'il importe d'obtenir.

La première condition est bien connue : le plan de l'image du disque solaire doit coïncider exactement avec la fente du collimateur; la seconde est non seulement négligée dans les spectroscopes, mais elle est même parfois rejetée comme défavorable à la beauté des images.

Cette condition est l'aplanétisme de l'image spectrale : elle consiste à faire coïncider, dans le plan du réticule de la lunette d'observation, les foyers des deux systèmes rectangulaires de lignes qu'on peut apercevoir dans le champ, à savoir les raies spectrales et les stries transversales causées par les défauts de la fente du collimateur (1). On reconnaît aisément que, si ces conditions sont remplies, le déplacement des faisceaux concentrés exactement dans le plan de la fente par la lentille collective est sans influence sur la position des raies spectrales, quelle que soit la loi de variation des intensités successives des faisceaux; tandis que, dans le cas con-

ne s’aurait trop s'attacher à réaliser la rigueur des conditions focales dont il est ici question pour obtenir sur les clichés des résultats probants.

Il est utile d'ajouter que l'intensité des radiations observées exerce une influence considérable sur la visibilité du phénomène : ainsi, dans les parties sombres du spectre solaire, la brisure et mème le balancement des raies deviennent de moins en moins perceptibles à mesure que l'éclat du champ diminue. Avec l'appareil dont je faisais usage, le phénomène s'effaçait au delà du groupe $B$ dans le rouge, en deçà de G dans le bleu. C'est alors que la photographie doit venir en aide à l'observateur.

( $\left.{ }^{1}\right)$ Dans le Mémoire détaillé, je donnerai la manière de corriger les spectroscopes de cette erreur d'aplanétisme, erreur généralement considérée comme avantageuse (comme dans les réseaux concaves), parce qu'elle débarrasse le champ de vision des strics horizontales, à peu près inévitables.

Il suffira de dire ici que cette correction s'obtient très aisément avec les réseaux, sans lentille auxiliaire, par la variation méthodique du tirage du collimateur et l'observation séparée des foyers des deux espèces de raies : les stries horizontales suivent la loi ordinaire des foyers conjugués, comme si le réseau n'existait pas; les raies spectrales sont soumises à une loi différente, de sorte qu’il est toujours possible de déterminer le plan où les deux systèmes de foyers coüncident.

Avec les spectroscopes à prismes, la correction sans lentille auxiliaire est moins simple : on l'obtient par une rotation convenable des prismes relativenent à la position du minimum de déviation. 
traire, il peut se produire un déplacement anormal, ou parallax" les images : de là l'impossibilité d'obtenir la fixité absolue des raies telluriques lors du balancement de la lentille collectrice. C'est probablement faute d'avoir eu égard à cette seconde condition que l'on n'a pas jusqu'ici obtenu cette fixité parfaite, nécessairc à la rigueur des résultats. Lorsque les plans de toutes les images focales remplissent bien la condition de coüncidence indiquée cidessus, la distinction des raies solaires et telluriques devient immédiate: on interroge, en quelque sorte individuellement, chaque raie; elle répond, par sa fixité, qu'elle est d'origine terrestre, par son balancement, qu'elle est d'origine solaire.

La figure ci-après met en évidence les principales raies d'origine solaire du groupe $\alpha$ (raies prolongées vers le bas) : presque toutes sont comparables en intensité aux raies telluriques voisines, ce qui produit la complexité apparente du premier abord. L'examen attentif de ces raies métalliques, sous une grande dispersion, montre que leur aspect est tout autre qu'on ne le croit généralement. Parmi les plus fortes, quelques-unes (telles que $\lambda=627,94$; $628,97 ; 629,84 ; 63 \circ, 03 ; 63 \circ, 13, \ldots)$ paraissent grises, larges et estompées sur les bords, caractère ordinaire des bandes d'absorption à froid; au contraire, les raies telluriques sont nettes, comme tracées au tire-ligne, et d'un noir très accentué. Cette sorte d'interversion des caractères ordinaires apportait une difficulté de plus à l'étude de cette région et, sans la méthode du balancement des raies, il eût fallu bien du temps pour éviter les confusions ( 1 ).

$2^{\circ}$ Raies dues à l'atmosphère sèche. - Le départ des raies d'origine solaire étant effectué, les principales raies telluriques qui subsistent (raies prolongées vers le haut, figure ci-dessus) forment

( $\left.{ }^{1}\right)$ Le Mémoire de M. Piazzi Smyth, Madeira spectroscopic, en fournit une preuve : les deux Planches (IX et X) consacrées à la comparaison du groupe $\alpha$, aux grandes et aux faibles hauteurs du Soleil au-dessus de l'horizon, présentent un relevé très détaillé de toutes les raies avec leurs intensités respectives dans les deux cas: malgré la variété et le nombre des observations qu'elles représentent la distinction des raies telluriques n'a pas été assez frappante pour mettre en évidence la symétrie de leur répartition, de sorte que l'analogie du groupe $\alpha$ avec $B$ et $A$ paraît avoir complètement échappé à l'éminent astronome royal d'Écosse. Ces deux Planches (II et $\mathrm{X}$ ) reproduisent fidèlement l'aspect complexe que présente le groupe $\alpha$ lorsqu'on l'examine pour la première fois. 
dcux séries inégales de doubles raies dont l'aspect cannelé rappelle
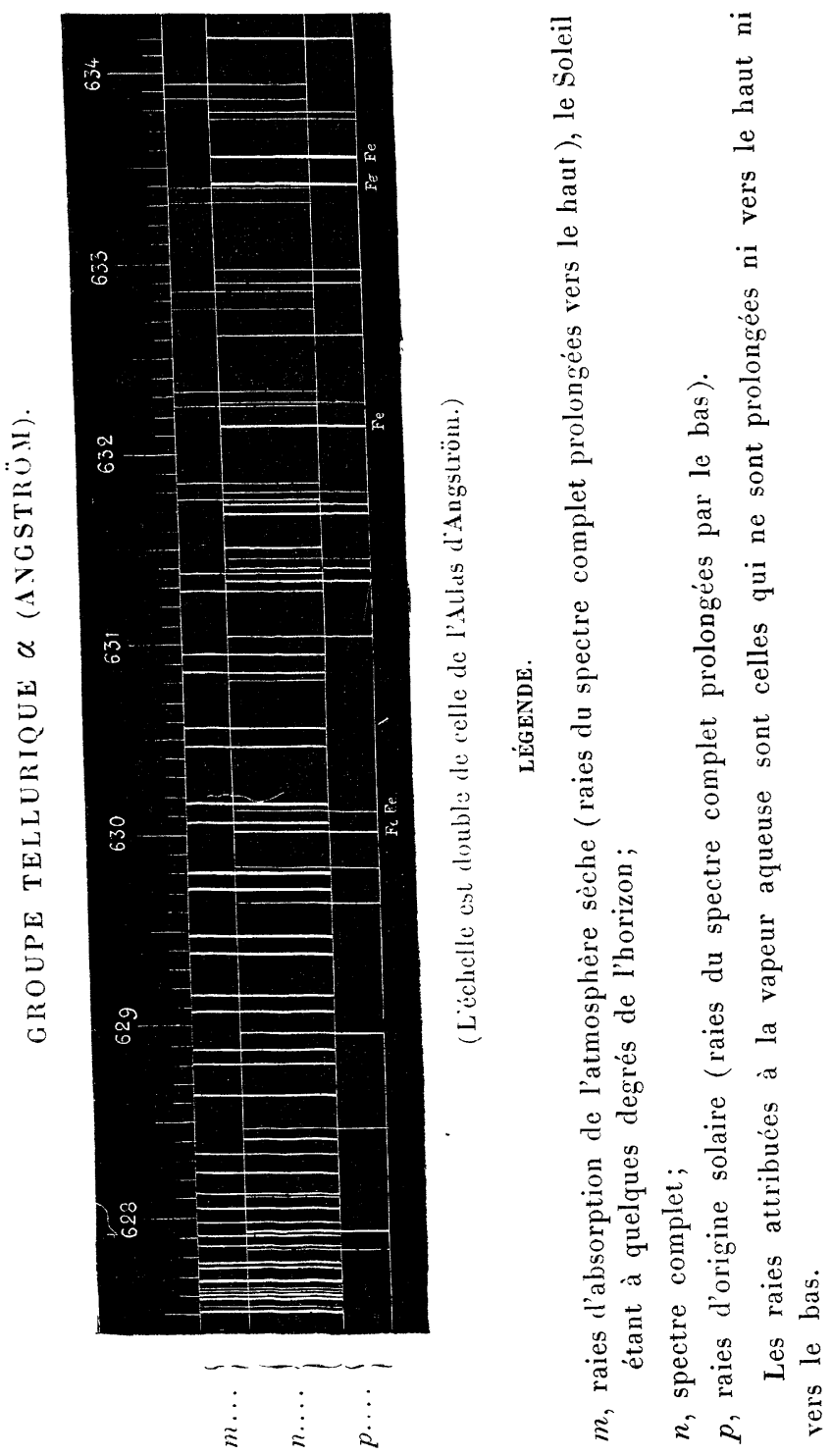

immédiatement celui des groupes telluriques A et B, si bien étudiés par M. Langley (Proceedings of the American Academy, 
$1878)$. Plus on examine ces trois groupes, plus l'analogie devienı frappante; on retrouve, en quelque sorte ligne pour ligne, les moindres détails de structure, si bien qu'on doit les considérer comme formant véritablement trois groupes harmoniques, analogues à ceux que présentent les spectres des éléments métalliques (doublets de l'aluminium, du calcium; triplets du magnésium, du zinc, du cadmium, etc.).

Cette identité de structure conduit forcément à admettre que le groupe $\alpha$ est produil par le même élément absorbant que A et B : c'est ce que pensait Angström, d'après ses observations hivernales; l'étude ci-dessus en constitue une véritable démonstration. La conséquence qu'on en déduit est fort importante : d'après des expériences directes, M. Egoroff (Comptes rendus, t. XCVII, p. 555) a annoncé que les groupes $\mathrm{A}$ et $\mathrm{B}$ doivent être attribués à l'absorption par l'oxygène de l'air (1). Il faut en conclure que $\alpha$ est également dû̀ l̀ l'oxygène. Comme l'intensité des groupes $\mathrm{A}, \mathrm{B}, \boldsymbol{\alpha}$ va en diminuant, on comprend comment l'auteur, ayant observé A d'abord, puis B, déjà très faible, n'a pu apercevoir $\boldsymbol{\alpha}\left({ }^{2}\right)$.

$3^{\circ}$ Raies de lavapeur aqueuse. - En dehors des raies solaires et de celles de la série précédente, on observe encore des raies

(') I. Piazzi Smyth (Madeira spectroscopic, p. 14) était, de son còté, arrivé à soupconner ce résultat : “ .....Je puis peut-ètre mentionner que j’ai récemment (novembre $\mathrm{r} 88 \mathrm{I}$ ) découvert une configuration des lignes du spectre de l'oxygène à basse température toutes différentes de celles de l'azote, mais ayant un grand air de famille avec les séries de lignes qu'on trouve dans ce mystérieux groupe $\alpha$ (a strong family resemblance to the bandelets of lines in this mysterious $\alpha$ band) " (Trans. R. Soc. Edinburgh, I880, 5i ). Toutefois, dans le spectre de l'oxygène publić par l'autea', il ne se trouve aucune raie coïncidant avec le groupe $\alpha$.

$\left({ }^{2}\right)$ Les inverses des longueurs d'onde des lignes homologues dans les trois bandes $\mathrm{A}, \mathrm{B}, \alpha$ sont à fort peu près en progression arithmétique : on est ainsi amené à prévoir la position approximative d'autres bandes complétant une série plus étendue. Dans l*infra-rouge, la Carte publiée par M. W' de W. Abney (Philosoph. Transactions of the Roy. Soc., 1880) montre en effet des groupes d'apparence très analogue; mais la définition des raies n'est pas assez parfaite pour qu'on puisse établir une concordance décisive.

Du côté du jaune, le calcul indique la possibilité d'une bande harmonique dans la position de la bande tellurique $\delta$; cette bande subsiste en effet par les plus grands froids; mais le groupe est si complexe et les raies sont si faibles que je n'ai pu encore parvenir à les ramener au type $\mathrm{AB} \alpha$. 
qui prennent aux basses hauteurs du Soleil une intensité considérable; elles ont un aspect particulier, qui les distinguerait à la rigueur des groupes précédents, mais leur caractère propre est de s'effacer presque entièrement lorsque l'atmosphère est froide et sèche : c'est ce que j'ai constaté définitivement le 24 janvier dernier, où le point de rosée s'est abaissé à $-3^{\circ}, 2$; dans le voisinage des raies $\mathrm{D}$, les raies telluriques avaient presque disparu; il est donc. naturel de les attribuer, comme les raies voisines de $\mathrm{D}$, à l'absorption causée par la vapeur d'eau. Les principales d'entre elles sont indiquées sur la figure par des lignes qui ne sont prolongées ni vers le haut, ni vers le bas $\left(\lambda=6{ }_{2} 8,13 ; 6{ }_{2} 8,44 ; 63\right.$ r, 5 I $)$.

Pour terminer, je ferai remarquer que la comparaison de ces diverses espèces de raies entre elles pourra conduire, ainsi que jai déjà eu l'occasion de l'exposer (Journal de l'École Polytechnique, LIII Cahier, p. 2 ıо, et Journal de Physique, $2^{\mathrm{e}}$ série, t. II, p. 63), à des résultats intéressant la Météorologie et l'Astronomie. Les raies du groupe $\alpha$ appartenant à l'atmosphère sèche auront l'avantage de présenter une échelle régulière d'intensité qui facilitera les mesures absolues.

En résumé, la présente étude du groupe $\alpha$ a conduit d'abord à une méthode pratique pour distinguer à la simple inspection les raies d'origine terrestre et celles d'origine solaire; elle a permis ensuite d'établir la relation intime de ce groupe avec les bandes A et $B$ de Fraunhofer; enfin elle a pour conséquence d'attribuer ce groupe à l'absorption par l'oxygène de l'air. 